\title{
Non-uniform Depth Compression Control Algorithm for 3D Real Time Video Detection
}

\author{
Wan Zhen-wu, Liu Lin-lin and Wang Ru*
}

Wuhan University of Technology Huaxia College, Wuhan Hubei, 430223, China

\begin{abstract}
For real-time stereo vision system, this paper analyzes the parallax generation mechanism of the virtual camera imaging; it presents projection transform of binocular images in single camera space. At the same time it changes the control depth perception by introducing camera wheelbase into bending deformation of the object, which can ensure the continuity of non-uniform compression ratio. The method is simple and flexible. The experimental results show that the depth mapping method will not visually produce distortion, which can effectively improve the quality of image. This method is a 3D graphics development for virtual reality and real-time game rendering, and it can be applied to 3D computer animation. When rendering stereo image, it can use this method to control the depth, which can reduce the amount of complicated work in post-production.
\end{abstract}

Keywords: Computer animation, depth mapping method, vision system, virtual camera, 3D graphics,

\section{INTRODUCTION}

Binocular stereo imaging technique can make the person produce stronger depth perception which can bring more realistic visual experience. Hence it is applied in many games, virtual reality production, film and other areas. In recent years, the stereo imaging technology has rapidly developed, but it still faces many challenges. One of the most critical problem is the long-term viewing $3 \mathrm{D}$ images will bring visual discomfort or fatigue, which is also the major factor restricting the development of stereoscopic film and game industry [1-4].

There are many factors tha cause visual fatigue, but a large number of research point that greater perceived depth range is one of the main reasons causing visual discomfort and visual fatigue [5-7]. When watching the stereoscopic images, there is a suitable depth range, as Fig. (1) shown. When the perceived depth is beyond this range, it will cause visual fatigue due to the conflict betiten human eye accommodation and binocular convergence $[1,6]$. Depth perception is related to binocular image disparity; therefore it can adjust the horizontal parallax of binocular image to control depth of scene perception within a suitable range, which is also known as a depth map [7, 8].

When adjusting the image disparity, it can directly adjust the two camera positions and parameters in the filming process, and it can also be adjusted by post processing. In the 3D film production, it often combines two ways [1]. In many real-time software systems (such as games, virtual reality and so on), it can only real-time adjust double camera parameters (such as the focal length, axial spacing) to compress depth perception $[9,10]$. Compressing sensing depth can reduce the visual discomfort, but it also can make the objects deformation in the scene (objects tend be to flat) [7, 11]. In order to reduce the contradiction betiten the two, a compromise is as far as possible keeping an important part of virtual scene intact [7].

For real-time stereo vision system, based on the projection transformation of virtual camera, this paper analyzes generation of image parallax and presents a simple, convenient depth mapping method. The method changes the image disparity through the object prior coordinate transformation, no need to change the camera's wheelbase at any time. The method can carry out continuous inhomogeneous depth mapping for the objects in the scene, using general mapping depth of virtual scene as restrictive conditions, it adopts different compression ratio for different parts to achieve a smooth depth compression, which can reduce the influence of depth compression on deformation.

\section{RELEVANT WORKING}

Binocular stereo display is the simulation of the human stereo vision formation mechanism [12]. People have done more research on the generation mechanism of visual fatigue $[3,6,13-15]$. A common view is: the human eye adjustment and convergence is a tightly coupling, but when watching the stereoscopic images, it will lead to the separation of adjustment and convergence. Large regulation conflicts cause visual discomfort or fatigue $[13,14]$. Patterson suggested in the production of stereo image, perceived depth should be limited within DOF (Depth of Field) [2]. Speranza and Wopking think the disparity threshold of suitable depth perception is about 1 degree $[4,5]$. From the adjusting convergence conflict and DOF perspective, in the shooting, if important content is put in the middle position on the screen (parallax is 


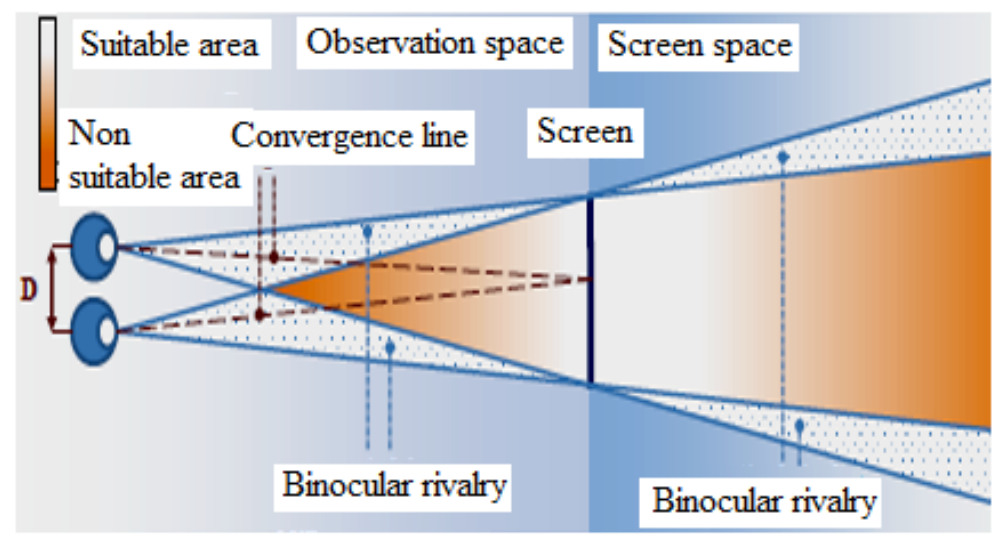

Fig. (1). The comfort zone of binocular viewing (the diagram is reference [1, 25]).

close to 0 ), it can further reduce the visual fatigue [1, 7]. Based on this idea, this paper gives the non continuous uniform depth mapping method, the contents near the screen remains smaller compression ratio.

It can be divided into two categories of methods for depth perception control: one is the method based on image synthesis; the other is to adjust the camera $\mathrm{g}$ wheelbase, focal length.

Based on original image, image synthesis produces binocular image which meets the new view point or a certain disparity, and computer vision theory provides a poitrful tool for this kind of method [16]. The classical method is using the depth map to calculate parallax, and then through the translation deformations of local image or the view interpolation, forming parallax images [17-21]. The depth information can be calculated from the left and right eye image disparity, and it also can obtain using the depth record camera $[22,23]$. A key problem of these methods encounter is as follows: it will exposure rear object originally in the change of disparity, thus forming the image empty, and empty redraw is still a challenging task. Another image synthesis method uses image deformation[24-27]. It avoids the production of image cavities, but it will image distortion. Experiments description of Kooi and Toet: non matching images will cause visual fatigue.

Image synthesis is mainly applied to the post processing of stereo image, sometimes it needs the help of complex manual operation, so it is difficult to be used in real-time rendering system. By adjusting the parameters of the camera in the shooting to achieve depth mapping is another method of optional. In order to reduce the workload of photographer, Zilly and Heinzle respectively develop the camera assisted automatic adjustment system, and it can real-time analyze the scene depth, camera focal plane, and adjust the parameters of the camera according to the requirement of shooting [28-30]. The control system is mainly used in practical camera. According to the real-time stereo vision system, Holliman and Oskam give depth mapping method of real time controlling the virtual camera $[7,8,10]$. In order to reduce the deformation of important contents, Holliman divide the scene into a plurality of regions, different regions use different compression ratio, but the compression ratio in different regions is not continuous $[7,8]$. In order to reduce the visual defect due to deformation, Sun gives a method of changing the depth compression rate according to general scene depth range, but when the camera moves it can lead to mutations in the depth range, and depth perception can occur mutation [9]. To solve this problem, Oskam has given a method of linear changing depth perception with time [10]. These non uniform depth compression methods are the segmented compression, and they use the same camera parameters at the same depth section. So there will be a catastrophe of compression deformation.

For real-time stereo vision system, based on the projection transformation of virtual camera, this paper analyzes generation of image parallax and gives a simple, convenient depth mapping method. This paper uses configuration parameters of the dual camera, in a single camera space, by using coordinate transformation generating binocular image. Parallax adjustment is changed into model vertex shift, which solves discontinuous problems in section compression.

\section{A SMOOTH NON UNIFORM MAPPING}

\subsection{Projection Transformation of Binocular image}

Because the horizontal deviation of stereo image is related to wheelbase of left and right camera and the deviation is a linear with the wheelbase. So the disparity calculation can be included in projection matrix. This paper uses projection matrix OpenGL as an example to derive the projection matrix of left and the right eye image. As shown in Fig. (2a), it sets an intermediate camera in the middle position of left and right eye camera $C_{\text {mid }}$, the distance of focal plane from the camera is $F$; camera wheelbase is $s ; h$ is the absolute offset of left camera relative to the right camera; $d$ is level absolute deviation of vertex $V$ relative to camera's projection. 


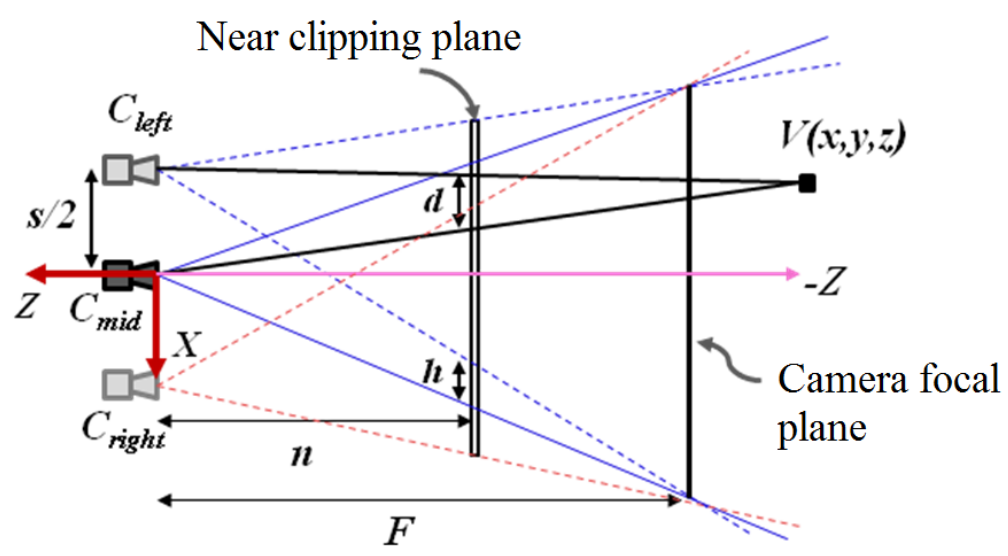

(a)

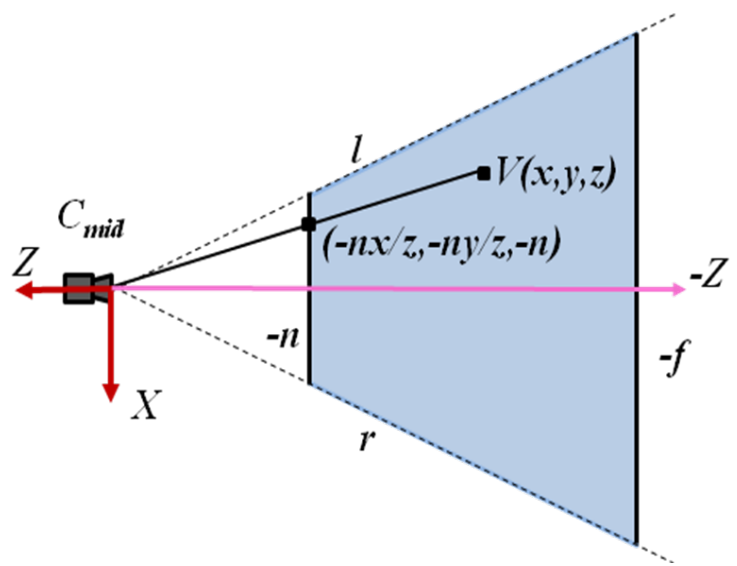

(b)

Fig. (2). Illustration of projection in camera space.

By the triangle geometry:

$h=\frac{F-n}{2 F} s$

$d=\frac{n+z}{2 z} s$

The viewing frustum of upper and loitr boundary for camera $C_{\text {mid }}$ is $t$ and $b$; the left and righ boundary are $l$ and $r$; far and near clipping plane are $-f$ and $-n$. According to Fig. (2b), in the $C_{\text {mid }}$ coordinate space it can get the projection coordinates of $V$ point in the $X$ direction.

$x_{m}=\frac{-n x}{z}$

Point coordinates corresponding to $\mathrm{x}$ on the left eye image is $x_{l}=x_{m}+h-d$. When the $V$ is after the focal plane, the value of $h-d$ is negative, which means the eye image shifts left relative to the center camera image, finally it will form the positive parallax. On the contrary, when the $V$ is before focus plane, the value of $h-d$ is negative, which means the eye image shifts right relative to the center camera image, finally it will form the negative parallax. Put the formulas (1), (2) and (3) into $x_{l}=x_{m}+h-d$.

$x_{l}=\frac{-n}{z} x+\frac{-n s}{2 F}-\frac{n s}{2 z}$

Because the binocular image exists only horizontal parallax, so it need only deal with the projection value of $x$ coordinate. Then the projection matrix of the left eye image is as follows:

$$
\left[\begin{array}{cccc}
\frac{2 n}{r-l} & 0 & \frac{n s}{F(r-l)}+\frac{r+l}{r-l} & \frac{n s}{r-l} \\
0 & \frac{2 n}{t-b} & \frac{t+b}{t-b} & 0 \\
0 & 0 & \frac{-(f+n)}{f-n} & \frac{-2 f n}{f-n} \\
0 & 0 & -1 & 0
\end{array}\right]
$$



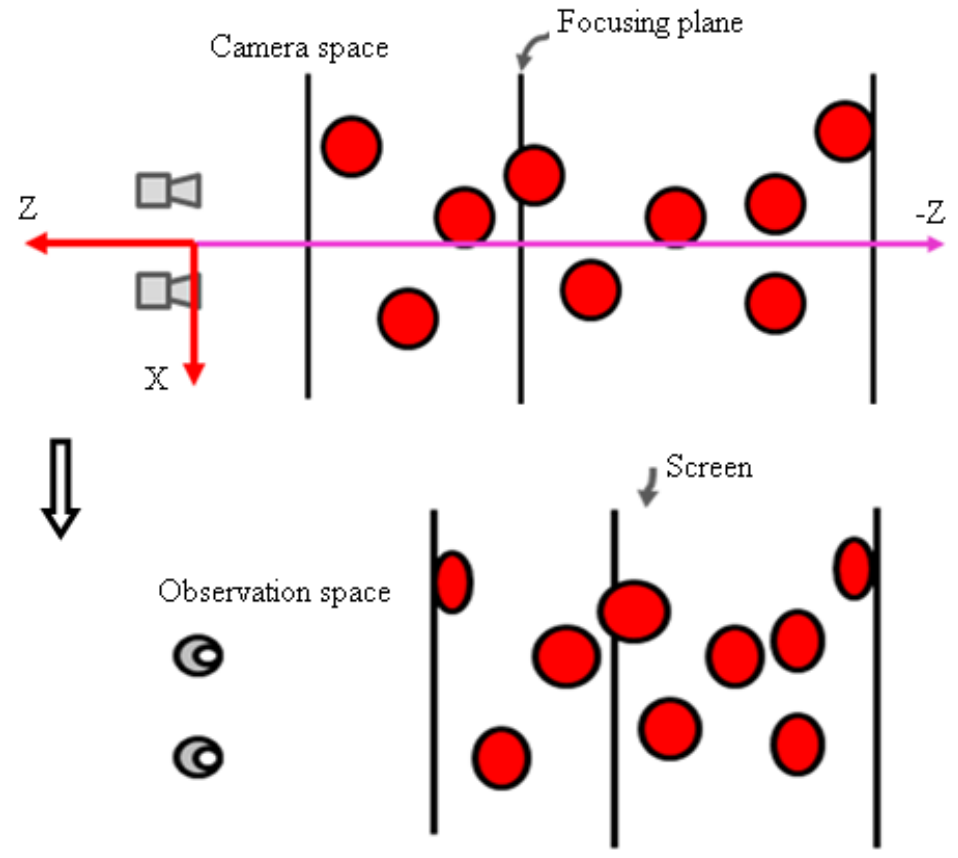

Fig. (3). Object deformation of non-uniform depth compression.

In general, the view frustum intermediate camera should be symmetric, $r=-l$ and $t=-b$.

$$
\left[\begin{array}{cccc}
\frac{n}{r} & 0 & \frac{n s}{2 F r} & \frac{n s}{2 r} \\
0 & \frac{n}{t} & 0 & 0 \\
0 & 0 & \frac{-(f+n)}{f-n} & \frac{-2 f n}{f-n} \\
0 & 0 & -1 & 0
\end{array}\right]
$$

Similarly, it can calculate the projection matrix of the right eye $C_{r p o j}$.

\subsection{Depth Compression}

Depth of compression will make the object deformation (tend to flat). According to Fig. (1), it know that the depth is the most appropriate near the screen position, human vision will pay more attention to the regions of the scene. So in order to reduce the defect caused by the deformation, it use non-uniform depth mapping to compress object of camera focusing planar at a given smaller ratio, and Fig. (3) shows the object far from the focus plane with big compression ratio.

According to the total limit depth perception and compression ratio, it can calculate the camera wheelbase of shooting scene at far, near clipping plane and the focal plane $[7,30]$. Assuming camera wheelbase is $S_{c}$ when shooting scene at the focus plane, camera wheelbases are $S_{f}$ and $S_{n}$ when shooting scene at far, near clipping plane. The camera wheelbase $S$ changing with vertices can be linear and nonlinear. Here, it give a linear transformation situation, the relationship betiten $S$ and $z$ is as shown in Fig. (4).

The relationship of $s-z$ is as follows:

$$
\left\{\begin{array}{l}
s=\frac{s_{c}-s_{f}}{f-F}(z+F)+s_{c},|z| \geq F \\
s=\frac{s_{c}-s_{n}}{n-F}(z+F)+s_{c},|z|<F
\end{array}\right.
$$

Input the formula (7) into formula (4), and convert it into a normalized device coordinates.

$$
\left\{\begin{aligned}
x_{\text {lclip }}= & \frac{-n}{z r}\left(x+\frac{f s_{c}-F s_{f}}{2(f-F)}+\frac{s_{c}-s_{f}}{2 F(f-F)} z^{2}\right. \\
& \left.+\frac{f s_{c}+F s_{c}-2 F s_{f}}{2 F(f-F)} z\right) \quad|z| \geq F \\
x_{\text {lclip }}= & \frac{-n}{z r}\left(x+\frac{\left(n s_{c}-F s_{f}\right)}{2(n-F)}+\frac{s_{c}-s_{f}}{2 F(n-F)} z^{2}\right. \\
& \left.+\frac{n s_{c}+F s_{c}-2 F s_{f}}{2 F(n-F)} z\right) \quad|z|<F
\end{aligned}\right.
$$

This is a nonlinear transform relationship, it is impossible to directly use matrix to transform. Before projection the vertex $V(x, y, z, 1)$ should contain $z^{2}$. it define a simple vector operation $\otimes$ to do coordinate transform on the vertices. Operational rules of $\otimes$ are as follows: 


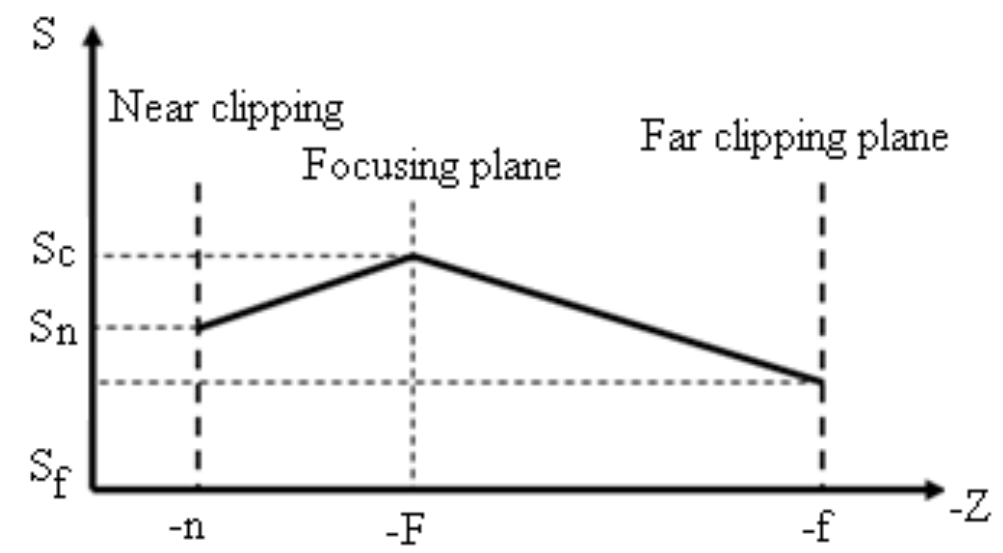

Fig. (4). The relation curve representing the inter-axis vary with the depth value $z$.

$$
\left[\begin{array}{l}
A \\
B \\
C \\
1
\end{array}\right] \otimes\left[\begin{array}{l}
x \\
y \\
z \\
1
\end{array}\right]=\left[\begin{array}{c}
x+A+B z+C z^{2} \\
y \\
z \\
1
\end{array}\right]
$$

Assuming a given transformation vector is $L(A, B, C)$, using formula (8) to do coordinate transform on vertices $V$, then using $S=S_{c}$ to do transform on projection matrix, so the equipment specification binocular image coordinates can be obtained, the transformation is:

$V_{\text {clip }}=M_{\text {proj }} \cdot(L \otimes V)$

Where $V_{\text {clip }}$ is the norms equipment coordinate after transformation, $M_{p r o j}$ is projection matrix of binocular image, here it need to determine the vector $L(A, B, C)$. If the transform vector corresponding to the left eye image is $L_{l}\left(A_{l}, B_{l}, C_{l}\right)$, so

$$
\left[\begin{array}{c}
x_{\text {lclip }} \\
y_{\text {lclip }} \\
z_{\text {lclip }} \\
-z
\end{array}\right]=\left[\begin{array}{cccc}
\frac{n}{r} & 0 & \frac{n s_{c}}{2 F r} & \frac{n s_{c}}{2 r} \\
0 & \frac{n}{t} & 0 & 0 \\
0 & 0 & \frac{-(f+n)}{f-n} & \frac{-2 f n}{f-n} \\
0 & 0 & -1 & 0
\end{array}\right] \cdot\left[\begin{array}{c}
x+A_{l} \\
B_{l} z+C_{l} z^{2} \\
y \\
z \\
1
\end{array}\right]
$$

Among them, $x_{l c l i p}=\left(x+B_{l} z+C_{l} z^{2}+\frac{s_{c}}{2 F} z+\frac{s_{c}}{2}+A_{l}\right) \frac{-n}{z r}$, then combined with equation 8 , it can obtain $A_{l}, B_{l}$ and $C_{l}$. When $|z| \geq F, A_{l}=\frac{F s_{c}-F s_{f}}{2(f-F)}, B_{l}=\frac{F s_{c}-F s_{f}}{F(f-F)}$ and $C_{l}=\frac{s_{c}-s_{f}}{2 F(f-F)}$; When $|z| \leq F, A_{l}=\frac{F s_{c}-F s_{n}}{2(n-F)}, B_{l}=\frac{F s_{c}-F s_{n}}{F(n-F)}$ and $C_{l}=\frac{s_{c}-s_{n}}{2 F(n-F)}$.
Similarly, it can get the transform vector of right eye image $L_{r}\left(A_{r}, B_{r}, C_{r}\right)$. The projection of left and right eye image can be expressed as:

$V_{\text {lclip }}=M_{l p r o j} \cdot\left(V \otimes L_{l}\right)$
$V_{\text {rclip }}=M_{\text {rproj }} \cdot\left(V \otimes L_{r}\right)$

Thus, in the generation of stereo image rendering process, it specify the boundary coordinates the camera of frustum $(f, n, t,-t,-r, r)$ and the focus plane distance $F$. According to the $(f, n)$ value and display equipment suitable observation depth range, it can calculate camera wheelbase $S_{f}, S_{n}$ and $s_{c}$. Further calculating $L_{l}$ and $L_{r}, M_{l p r o j}$ and $M_{r p r o j}$ when $s=s_{c}$. When rendering, the use of formula (12) can generate binocular image.

\subsection{Analysis}

Stereoscopic image generating method is based on single camera space, by using the projection matrix respectively generating left and right images, it is simple than double camera. In fact, the matrix in formula (1) can be further separated into two matrix multiplication:

$$
M_{l p r o j}=M_{c p r o j} \cdot M_{l p a r}\left[\begin{array}{cccc}
\frac{n}{r} & 0 & 0 & 0 \\
0 & \frac{n}{t} & 0 & 0 \\
0 & 0 & \frac{-(f+n)}{f-n} & \frac{-2 f n}{f-n} \\
0 & 0 & -1 & 0
\end{array}\right] \cdot\left[\begin{array}{cccc}
1 & 0 & \frac{s}{2 F} & \frac{s}{2} \\
0 & 0 & 0 & 0 \\
0 & 0 & 1 & 0 \\
0 & 0 & 0 & 1
\end{array}\right]
$$

The first matrix $M_{c p r o j}$ is standard projection matrix in OpenGL, the second matrix $M_{\text {lpar }}$ is used to generate image horizontal parallax. Similarly it can calculate the disparity matrix of right eye $M_{r p a r}$. 

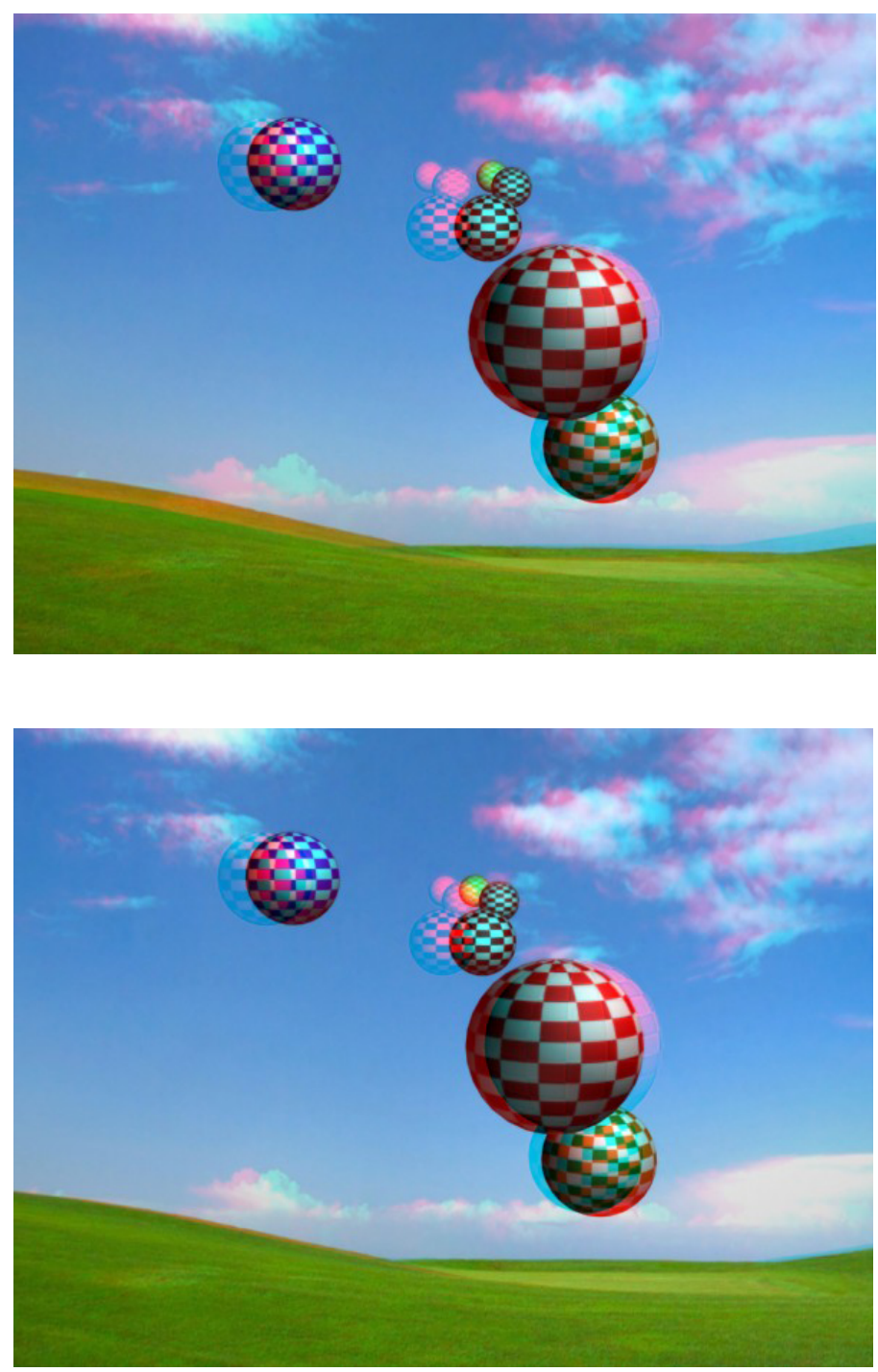

(a)

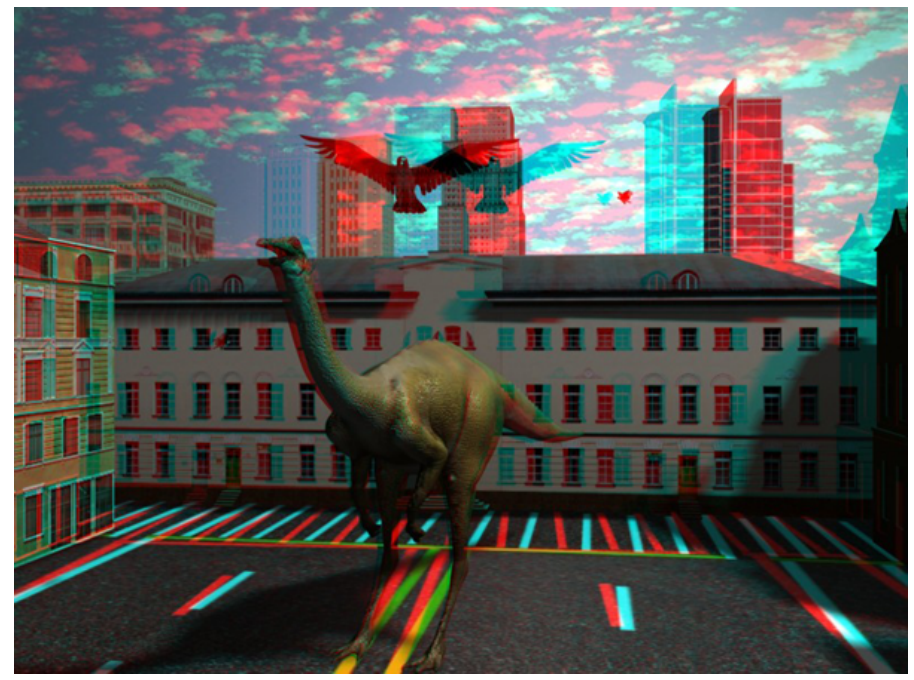

Fig. (5). Contd... 


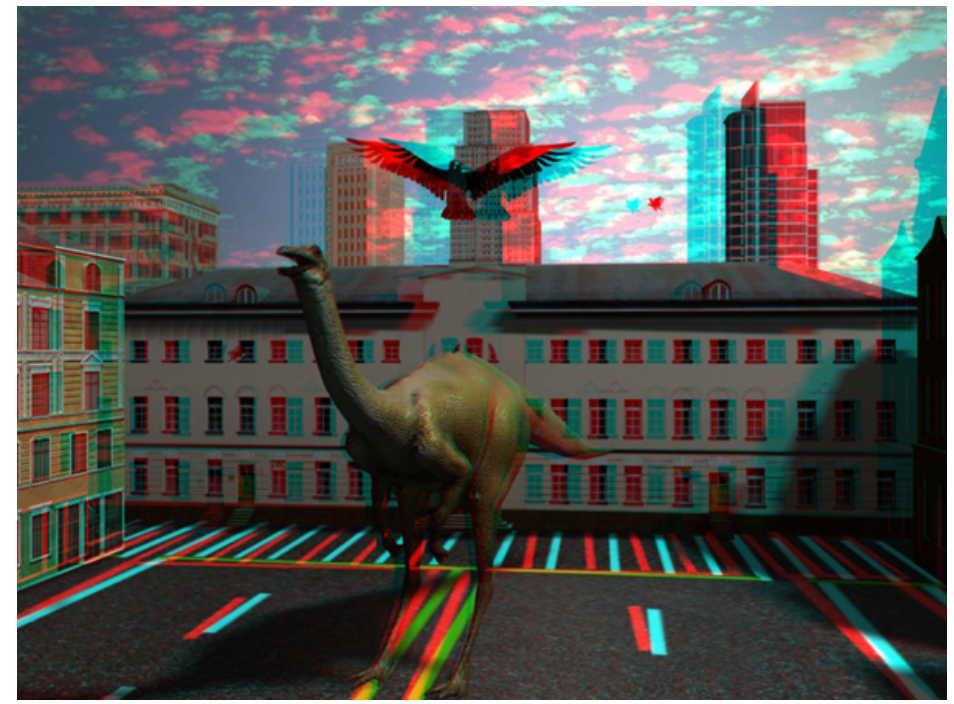

(b)

Fig. (5). Results of the depth compression using our method. The left are the original images before depth compression and the right are the stereoscopic images after depth compression.

$$
M_{r p a r}=\left[\begin{array}{cccc}
1 & 0 & -\frac{s}{2 F} & -\frac{s}{2} \\
0 & 0 & 0 & 0 \\
0 & 0 & 1 & 0 \\
0 & 0 & 0 & 1
\end{array}\right]
$$

$M_{\text {lpar }}$ and $M_{r p a r}$ are shear transformation matrix. Therefore, the formation of binocular images can be vieitd as the shear transformation in single camera space, and then the conventional projection, finally it can see that shear transformation is symmetric.

$\otimes$ operation is a nonlinear transformation, through the transformation, it can change the size of right and left eyes image parallax and realize the depth mapping. $\otimes$ transformation is bending deformation on the $z$ axis. In the example given in this paper, the wheelbase is linear variation with depth $z$, so the model deformation curve is $x=A+B z+C z^{2}$. So the drawing process of the whole stereo image can also be understood as: first performed bending deformation on the model, then shear transformation, finally the projection imaging. Binoculars bending deformation is symmetric, $L_{l}=(A, B, C)$ and $L_{r}=(-A,-B,-C)$.

\section{EXAMPLE ANALYSIS}

In Fig. (5), it uses the results of depth map that can be seen the stereo effect by using red-green glasses, the left is picture before compression, the right is result after compression. In Fig. (5a), the front of red ball and the green ball are in focus plane nearby, so the depth changes are small, but with the increases of compression ratio, the last side of the ball tends to move forward; In the left diagram of Fig. (5b), the flying eagles because too large disparity, it produces diplopic phenomenon (binocular rivalry), it will disappear after compression, but the nearby dinosaurs in the focusing plane do not happen too much change.

As previously mentioned, it are through the bending deformation on the scene to adjust parallax, then along the line of sight to place narrow object, whether or not to see the object bending phenomenon after depth mapping. Fig. (6) shows the results of a long drawn cuboids monocular observations can see cuboids have bent, but in the case of stereoscopic observation but do not see curved phenomenon. This is because in the acquisition of binocular image, corresponding to the two eyes of deformation object is smooth symmetry, and only in the horizontal direction bending, in the binocular observation, this bending only reflects the continuous change of parallax. Therefore, the use of smoothing it give non-uniform depth mapping method, the shape of the object in addition to generate depth decreases, the deformation will not produce the other direction.

Unlike other piecewise inhomogeneous compression method, the given smooth variable proportion depth compression method does not require section to obtain scene images, so the implementation is more convenient $[7,8,10]$. This paper only gives the examples of camera wheelbase linearly varies along the depth, for the nonlinear complicated situations it can realize, it only need to compute the vector $L$ of bending transformation, this calculation is very easy to implement, but $L$ may be a more than three dimensional vector, then it can redefine " $\otimes$ " operation.

\section{SUMMARY}

In order to reduce visual fatigue caused by excessive horizontal disparity in stereo images, this paper investigates the real-time rendering of stereo vision system, and presents a 


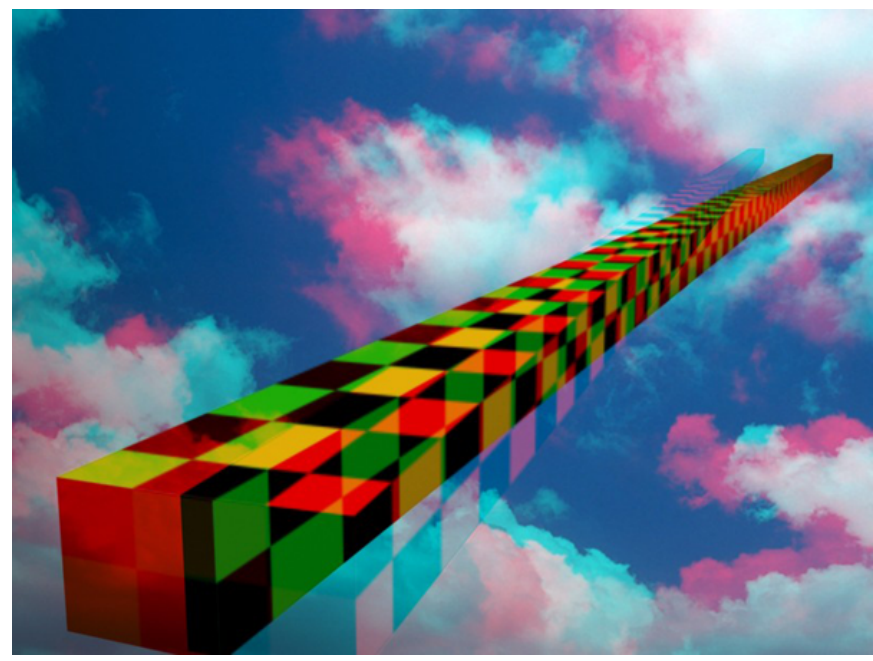

(a) The original images before depth compression

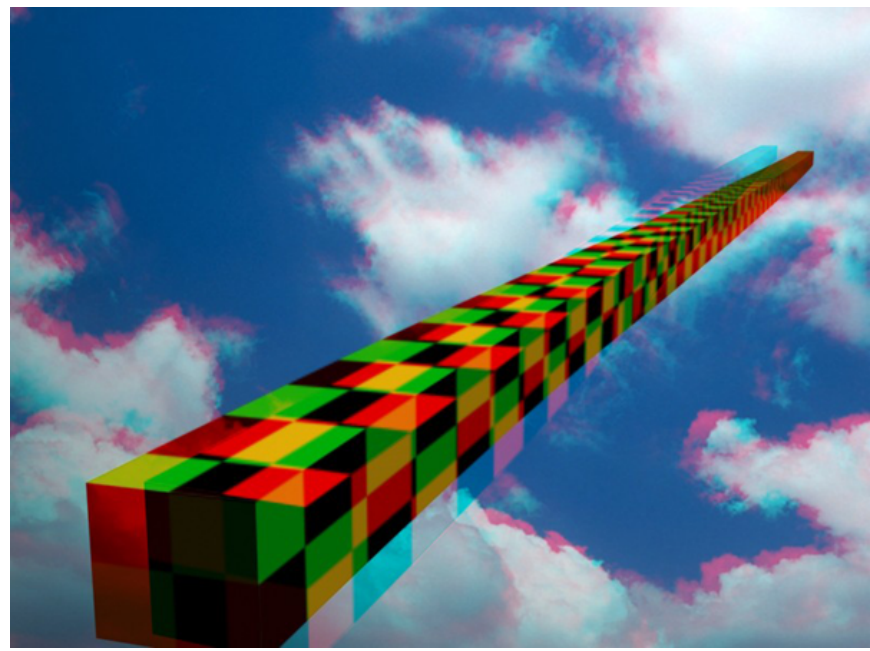

(b) The stereoscopic images after depth compression

Fig. (6). Comparision betiten the stereoscopic images of non-compression and compression.

non-uniform depth compression method. In the single camera space, the method can not only generate binocular image through the coordinate transformation, but also go through coordinate transformation to control the horizontal parallax. In order to reduce model deformation flaws caused by the depth of compression, the objects of different depth region carries out different compression ratio, and the camera wheelbase is expresses as continuous function for depth, it can go through the camera wheelbase to derive the coordinate transformation of binocular image in a single camera space, so as to ensure the continuous change of the compression ratio. The experimental results show that this method can effectively improve the quality of stereo image, which is not only simple and effective, but also can be applied in game, virtual reality and other real-time stereo vision system.

\section{CONFLICT OF INTEREST}

The authors confirm that this article content has no conflict of interest.

\section{ACKNOWLEDGEMENTS}

Declared none.

\section{REFERENCES}

[1] B. Mendiburu, 3D Movie Making, Elsevier Press, Amsterdam, 2009.

[2] R. Patterson, "Review paper- human factors of stereo displays-an update," Journal of the Society for Information Display, vol. 17, no. 12, pp. 987-996, 2009.

[3] M. Lambooij, F. Marten, H. Ingrid, and W. Jsselsteijn, "Visual discomfort and visual fatigue of stereoscopic displays-a review," Journal of Imaging Science and Technology, vol. 53, no. 3, pp. 114, 2009.

[4] F. Speranza, W.J. Tam, R. Renaud, and N. Hur, "Effect of disparity and motion on visual comfort of stereoscopic images," In: Proceedings of SPIE 6055, Stereoscopic Displays and Virtual Reality Systems XIII, Bellingham, WA: SPIE Press, Article no. 60550B, 2006.

[5] M. Wopking. "Viewing comfort with stereoscopic pictures: an experimental study on the subjective effects of disparity magnitude and depth of focus," Journal of the Society for Information Display, vol. 3, no. 3, pp. 101-103, 1995. 
[6] T. Shibata, J. Kim, D. M. Hoffman, and M. S. Banks, 'The zone of comfort: predicting visual discomfort with stereo displays," Journal of Vision, vol. 11, no. 8, pp. 1-29, 2011.

[7] N. S. Holliman, "Mapping Perceived Depth to Regions of Interest in Stereoscopic Images," In: Proceedings of SPIE 5291, Displays and virtual reality systems XI, Bellingham, WA: SPIE Press, 2004, pp. $117-128$

[8] N. S. Holliman, "Smoothing region boundaries in variable depth mapping for real-time stereoscopic images," In: Proceedings of the SPIE 5664, Bellingham, WA:SPIE Press, 2005, pp. 281-292.

[9] G. Sun, and N. Holliman, "Evaluating methods for controlling depth perception in stereoscopic cinematography," In: Proceedings of the SPIE, Stereoscopic Displays and Applications, Bellingham, WA: SPIE Press, vol. 7237, 2009, doi: 10.1117/12.807136; http://dx.doi.org/10.1117/12.807136.

[10] T. Oskam, A. Hornung, H. Bowles, K. Mitchell, and M. Gross "OSCAM- optimized stereoscopic camera control for interactive 3D," In: Proceedings of ACM SIGGRAPH Asia (Hong Kong, China, December 12-15, 2011), ACM Transactions on Graphics, vol. 30, no. 6, pp. 1-8, 2011.

[11] Z. Wartell, L. F. Hodges, and W. Ribarsky, "Balancing fusion, image depth and distortion in stereoscopic head- tracked displays," In: Proceeding of SIGGRAPH'99, New York: ACM Press, 1999, pp.351-358.

[12] S. Reichelt, R. Häussler, G. Fütterer, and N. Leister, "Depth cues in human visual perception and their realization in 3D displays," In: Proceedings of SPIE 7690, Display Technologies and Applications for Defense, Security, and Avionics IV, 7690B, Bellingham, WA: SPIE Press,: Article NO.76900B, 2010.

[13] D. M. Hoffman, A. R. Girshick, K. Akeley, and M. S. Banks, "Vergence- accommodation conflicts hinder visual performance and cause visual fatigue," Journal of Vision, vol. 3, no. 3, pp. 1-30, 2008.

[14] M. Emoto, T. Niida, and F. Okana, "Repeated vergence adaptation causes the decline of visual functions in watching stereoscopic television," Journal of Display Technology, vol. 1, no. 2, pp. 328-340, 2005.

[15] Y. Okada, K. Ukai, J.S. Wolffsohn, B. Gilmartin, A. Iijima, and T. Bando "Target spatial frequency determines the response to conflicting defocus- and convergence-driven accommodative stimuli," Vision Research, vol. 46, no. 4, pp. 475-484, 2006.

[16] A. Smolic, P. Kauff, S. Knorr, A. Hornung, M. Kunter, M. Muller, and M. Lang, "Three-Dimensional Video Postproduction and Processing," Proceedings of the IEEE, vol. 99, no. 4, pp. 607-625, 2011.

[17] L. Zhang, and J.T. Wa, "Stereoscopic Image Generation Based on Depth Images for 3D TV," IEEE Transactions on Broadcasting, vol. 5, no. 2, pp. 191-199, 2005 .
[18] A. Smolic, K. Mller, K. Dix, P. Merkle, P. Kauff, and T. Wiegand, "Intermediate view interpolation based on multiview video plus depth for advanced 3D video systems," In: Proceedings of $15^{\text {th }}$ IEEE International Conference on Image Processing, Los Alamitos: IEEE Computer Society Press, 2008, pp. 2448-2451.

[19] C.L. Zitnick, S.B. Kang, M. Uyttendaele, S. Winder, and R. Szeliski, "High-quality video view interpolation using a layered representation,' ACM Transaction on Graphics, vol. 23, no. 3, pp. 600-608, 2004

[20] L. Zhang, D. Wang, and A. Vincent, "Adaptive reconstruction of intermediate views from stereoscopic images", IEEE Transaction on Circuits System for Video Technology, vol. 16, no. 1, pp. 102113,2006

[21] M. Xi, L.H. Wang, Q.Q. Yang, D.X. Li, and M. Zhang "Depthimage-based rendering with spatial and temporal texture synthesis for 3DTV," EURASIP Journal on Image and Video Processing, vol. 9, pp. 1687- 5281, 2013.

[22] H. Hirschmueller, "Stereo vision in structured environment by consistent semi-global matching," In: Proceedings of IEEE CVPR'2006, Los Alamitos: IEEE Computer Society Press, 2006, pp. 2386-2393.

[23] B. Bartczak and R. Koch, "Dense depth maps from low resolution time-of-flight depth and high resolution color views," Lecture Notes in Computer Science. Heidelberg: Springer, vol. 5876, pp. 228-239, 2009

[24] Y. Z. Niu, W. C. Feng, and F. Liu, "Enabling warping on stereoscopic images," ACM Transaction on Graphics, vol. 31, no. 6, Article No. 183, 2012.

[25] M. Lang, A. Hornung, O. Wang, S. Poulakos, A. Smolic, and M. Gross "Nonlinear disparity mapping for stereoscopic 3D," $A C M$ Transaction on Graphics, vol. 29, no. 4, Article no. 75, 2010.

[26] T. Yan, R.W.H. Lau, Y. Xu, and L.S. Huang, "Depth mapping for stereoscopic videos," International Journal of Computer Vision, vol. 102, no. 1-3, pp. 293-307, 2013.

[27] B. Masia, G. ittzstein, C. Aliaga, R. Raskar, and D. Gutierrez, "Display adaptive 3D content remapping," Computers \& Graphics, vol. 37, no. 8, pp. 983-996, 2013.

[28] F.L. Kooi, and A. Toet, "Visual comfort of binocular and 3-D displays," Displays, vol. 25, no. 2-3, pp. 99-108, 2004.

[29] F. Zilly, M. Müller, P. Eisert, and P. Kauf, "The stereoscopic analyzer-an image-based assistance tool for stereo shooting and 3D production," In: Proceedings of $17^{\text {th }}$ IEEE International Conference on Image Processing, Los Alamitos: IEEE Computer Society Press, 2010, pp. 4029-4032.

[30] S. Heinzle, P. Greisen, and D. Gallup, "computational stereo camera system with programmable control loop," ACM Transaction on Graphics, vol. 30, no. 4, Article No. 94, 2011.

\author{
Received: September 16, 2014 \\ Revised: December 23, 2014 \\ Accepted: December 31, 2014 \\ (C) Zhen-wu et al.; Licensee Bentham Open.
}

This is an open access article licensed under the terms of the Creative Commons Attribution Non-Commercial License (http://creativecommons.org/licenses/by$\mathrm{nc} / 3.0 /$ ) which permits unrestricted, non-commercial use, distribution and reproduction in any medium, provided the work is properly cited. 